\title{
Quasi-one-dimensional self-assembly of metal nanoclusters on C/W(110)
}

\author{
Magdalena Bachmann, Martin Gabl, Clemens Deisl, Norbert Memmel, ${ }^{*}$ and Erminald Bertel \\ Institute of Physical Chemistry, University of Innsbruck, Innrain 52a, A-6020 Innsbruck, Austria
}

(Received 8 September 2008; published 3 December 2008)

\begin{abstract}
Quasi-one-dimensionally ordered chains of silver and cobalt clusters are grown on the $R(15$ $\times 12)-\mathrm{C} / \mathrm{W}(110)$ template and investigated by scanning-tunneling microscopy. Both Ag and Co nucleate at the same area within the large template unit cell. We attribute this area to the carbon-poor part of the unit cell. Clusters exhibit a narrow size distribution, peaking at cluster sizes of seven to eight atoms. The strong preference of this cluster size is attributed primarily to the size of favorable adsorption areas in the $R(15$ $\times 12$ ) unit cell. For cobalt atoms the adsorption strength seems to be more homogeneous across the unit cell than for $\mathrm{Ag}$, allowing also growth of small clusters on less favorable regions of the unit cell at low temperatures.
\end{abstract}

DOI: 10.1103/PhysRevB.78.235410

PACS number(s): 81.16.Dn, 36.40.Sx, 61.46.Bc, 68.65.Cd

\section{INTRODUCTION}

Several applications of nanoscience and nanotechnology require large-scale fabrication of nanosized structures, such as nanodots or nanowires. ${ }^{1}$ Formation of well-ordered arrays of nano-objects is often required to ensure the functionality of such structures, e.g., in microelectronics, ultrahigh-density recording, or as antennas to confine light to subwavelength regions. ${ }^{2}$ Since top-down production methods are expensive, time-consuming, and often limited to sizes well above 20-50 $\mathrm{nm}$, bottom-up fabrication by self-assembly is considered to be a promising alternative.

One of the most widely used processes for the selfassembly of well-ordered cluster arrays is deposition on prestructured template surfaces. The idea behind this method is that the template defines a periodic array of preferred adsorption sites, from which the clusters start to nucleate. Surface structures of various origins have been used as predefined nucleation sites, e.g., surface lattice dislocations, surface reconstructions, vicinal surfaces, Moire patterns, adsorbate superstructures, or combinations thereof. ${ }^{3-14}$

In addition to the regular arrangement, a narrow size and shape distribution of the deposited clusters is required in order to guarantee identical behavior of all nano-objects. In the ultrasmall regime $(\$ 2 \mathrm{~nm})$, size and shape of nanoclusters ultimately have to be specified with atomic precision as addition or removal of a few atoms may already strongly alter the cluster properties. In this respect the preferred occurrence of particularly stable clusters, so-called "magic" clusters, has gained special attention. For surface-supported clusters, the stability depends on the interplay between adsorbate-adsorbate and adsorbate-substrate interactions. ${ }^{7,15}$ If adsorbate-substrate interactions are weak and are sufficiently uniform over the surface, cluster formation is dominated by adatom-adatom interactions and magic cluster sizes as in the case of free unsupported two-dimensional clusters can be observed. ${ }^{9}$ In contrast, if adsorbate-substrate interactions are strong and if the potential landscape of the template is strongly inhomogeneous, the width of the attractive regions of the template potential minima will render the growth of larger objects more difficult. As a consequencesufficient adatom mobility provided-a sharp cluster size distribution will be obtained, in particular close to the "ideal" coverage, defined as the coverage where all potential minima are completely filled with clusters.

In the present work we introduce a template- the $R(15$ $\times 12)$-C/W(110) surface-for the ordered deposition of metal nanoclusters of both noble $(\mathrm{Ag})$ and transition metals (Co). We provide evidence that — as described above — both position and size of the clusters are governed by adsorbatesubstrate interactions and are predefined by the potential landscape of the template. Clusters are arranged on the $R(15 \times 12)$ rectangular grid of $1.37 \times 3.10 \mathrm{~nm}^{2}$. Due to the anisotropic grid, quasi-one-dimensional cluster chains can be fabricated close to the ideal coverage. The clusters exhibit a narrow size distribution, favoring a size of seven to eight atoms. We determine the optimum preparation conditions and the cluster adsorption site within the $(15 \times 12)$ unit cell for both deposited metals, and discuss the origin of the cluster stability and long-range order as well as similarities and differences between both metal types.

\section{EXPERIMENT}

Experiments were carried out in an UHV system (base pressure of $1 \times 10^{-10} \mathrm{mbar}$ ), equipped with a Danish Micro Engineering (DME) room-temperature scanning-tunneling microscope (STM). Sample temperatures were monitored with either a two color or an optical pyrometer at high temperatures and an infrared (IR) pyrometer at temperatures below $1000 \mathrm{~K}$. Temperature readings were estimated to be accurate within $\pm 50 \mathrm{~K}$. Routine cleaning of $\mathrm{W}(110)$ was achieved by flashing to $2300 \mathrm{~K}$, annealing at $1550 \mathrm{~K}$ in 5 $\times 10^{-8}$ mbar oxygen, and a final flash to $2300 \mathrm{~K}$.

Starting from a clean surface, the $R(15 \times 12)-\mathrm{C} / \mathrm{W}(110)$ system was prepared by thermolysis of ethene at a pressure of $5 \times 10^{-8}$ mbar and temperatures of $1250-1700 \mathrm{~K}$, followed by flashing two times to $2300 \mathrm{~K}$ with subsequent rapid cooling $(\approx 70 \mathrm{~K} / \mathrm{s})$. If the sample already contained sufficient carbon, the deposition step was omitted, and the $(15 \times 12)-\mathrm{C} / \mathrm{W}(110)$ structure was prepared by flashing and cooling cycles alone.

$\mathrm{Ag}$ was deposited from a resistively heated alumina crucible. Deposition rates ranged from 0.03 to 0.3 monolayers 
$(\mathrm{ML}) / \mathrm{min}$. Cobalt was evaporated from a $2 \mathrm{~mm}$ rod using $e$-beam heating and deposition fluxes between 0.6 and 1.2 $\mathrm{ML} / \mathrm{min}$. Co ions generated by the $e$-beam bombardment were electrostatically deflected out of the deposition beam. Coverages were deduced from deposition times and calibrated from STM images by evaluating the Ag or Co areas, respectively, under conditions were large islands were formed. Coverages were found to be reproducible with an accuracy of approximately 10\%-15\%. Metal deposition was performed during down cooling after the final flash in the template preparation sequence, resulting in a slight temperature decrease during deposition.

\section{RESULTS AND DISCUSSION}

\section{A. $R(15 \times 12)-\mathrm{C} / \mathrm{W}(110)$ Template}

The $R(15 \times 12)-\mathrm{C} / \mathrm{W}(110)$ structure was reported for the first time in the low-energy electron diffraction (LEED) investigations of Bauer, ${ }^{16}$ as well as Baudoing and Stern. ${ }^{17}$ If only little carbon is present in the bulk of the tungsten crystal, it can be prepared out of the high-coverage carbon superstructure- $R(15 \times 3)-\mathrm{C} / \mathrm{W}(110)$ - by annealing to $2300 \mathrm{~K}$ (to dissolve carbon partially into the bulk) and rapid down cooling (to avoid segregation of carbon back to the surface). Note that the notations of both carbon superstructures were used for historical reasons and do not follow the standard Wood nomenclature. ${ }^{18}$ For the $R(15 \times 12)$ structure a correct Wood notation would be $\left(\frac{5}{2} \sqrt{3} \times 4 \sqrt{3}\right) R 54.7^{\circ}$ relative to the conventional centered rectangular unit cell of the $\mathrm{W}(110)$ surface or $\left(\begin{array}{cc}5 & 0 \\ -4 & 12\end{array}\right)$ in the Park-Madden matrix notation relative to the primitive basis vectors $\mathbf{a}_{1}=\frac{1}{2}(1 \overline{1} 1)$ and $\mathbf{a}_{2}=\frac{1}{2}(1 \overline{1} \overline{1})$. The $R(15 \times 12)$ unit cell is shown in Fig. 1(b). It covers an area equivalent to $60 \mathrm{~W}$ atoms of an unreconstructed W(110) surface. Due to the mirror symmetry of the $\mathrm{W}(110)$ substrate, there exist two domains with the short unit-cell axes oriented along [1 $1 \overline{1} 1]$ and [ $\overline{1} 11]$, respectively.

The atomic configuration within the large unit cell has not been determined so far. Even the carbon concentration is not known exactly. According to the segregation analysis of Foulias et al., ${ }^{19}$ the $\mathrm{C}$ concentration is about a factor of three lower than for the high-coverage $R(15 \times 3)$ phase. For the latter, Auger electron spectroscopy (AES) data ${ }^{20}$ as well as kinetic adsorption data ${ }^{21}$ yield a coverage of $\approx 0.63 \mathrm{ML}$, whereas from the interpretation of atomically resolved STM images $^{22,23}$ a structure model with $0.4 \mathrm{ML}$ was suggested. Thus the carbon concentration for the $R(15 \times 12)$ is expected to be in the range of $0.13-0.21 \mathrm{ML}$.

In most cases STM images of the $R(15 \times 12)$ structure show a characteristic unit-cell pattern with one bright and two small protrusions along [1 $1 \overline{1} 2]$ or [ $\overline{1} 12]$, respectively, ${ }^{22,13}$ as it was also observed in the present work [Fig. 1(a)]. STM images of Bode et al. ${ }^{22}$ taken at low tunneling resistance exhibit a lens-shaped hollow, surrounded by protruding atomically resolved areas exhibiting the same periodicity as the W(110) substrate. From this observation the authors concluded that carbon induces a "periodic buckling of the surface while leaving the crystal structure of the W(110) substrate unaffected in parts of the unit cell," which also implies that these parts of the unit cell are almost free of carbon. Although the protruding regions in low and high tunneling resistance images are not necessarily the same, it is tempting to associate the bright protrusions in Fig. 1(a) with the carbon-poor "clean" parts of the $(15 \times 12)$ unit cell. This supposition will be further substantiated by the Ag adsorption data described below.

In the STM images of Figs. 3(a) and 5(a) taken at low Ag and Co coverages on the $R(15 \times 12)$ template, a somewhat different $R(15 \times 12)$ pattern than that in Fig. 1(a) was observed. The dominating features in Fig. 5(a) are rows of depressions (one of them marked by an arrow) separated by short bright stripes oriented at an angle of $\approx 70^{\circ}$ relative to

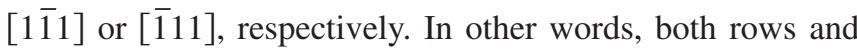
short stripes are oriented along close-packed directions of the substrate. The same pattern-however less pronounced-can be recognized in Fig. 3(a) [see region encircled in yellow in the top part of Fig. 3(a)]. In addition, between the depressions of neighboring rows, two faint maxima are observed [arrows in the inset of Fig. 3(a)]. We attribute the depressions of Figs. 3 and 5 to the same areas of the unit cell as the bright protrusions in Fig. 1(a) for three reasons: (i) close inspection of the STM data in Refs. 13 and 22 reveals that some of the bright protrusions already show indications of a dip in their center. Under the tunneling conditions of Figs. 3 and 5, these "dips" developed into the pronounced center holes. As the images of Figs. 3(a) and 5(a) (which show depressions) were obtained at quite different tunneling resistances $(U=30 \mathrm{mV}, I=0.58 \mathrm{nA}$, and $R=52 \mathrm{M} \Omega$ vs $U$ $=1100 \mathrm{mV}, I=0.10 \mathrm{nA}$, and $R=11 \mathrm{G} \Omega$ ) and Fig. 1(a) (which exhibits the bright protrusions) was taken with intermediate tunneling resistance $(U=173 \mathrm{mV}, I=0.28 \mathrm{nA}$, and $R=620 \mathrm{M} \Omega$ ), we concluded that the different appearance of the $R(15 \times 12)$ superstructure was primarily related to different tip conditions rather than electrical tunneling parameters. (ii) The two faint maxima between the main features of neighboring rows are observed in both types of images [see also inset of Fig. 3(a)]. (iii) Occasionally out-of-phase domain boundaries appear, resulting in row spacings increased by $\approx 0.7$ or $\approx 1.4 \mathrm{~nm}$, respectively. In these cases one or two additional faint protrusions appear in both types of images. The distances between the faint protrusions are the same as in the case of the regular row spacing while the distance between the main features [bright protrusions as in Fig. 1(a) or depressions as in Figs. 3(a) and 5(a), respectively] increases with the row distance. In the upper left part of Fig. 1(a) and the right part of Fig. 3(a), such increased row spacings can be discerned.

Even for our most perfect preparations of the $(15 \times 12)$ structure, we found regions on the samples that do not exhibit the $R(15 \times 12)$ structure. We attribute these regions to clean tungsten patches still present on the surface. As discussed in more detail in the next chapter, these patches are best discriminated from the $R(15 \times 12)$ regions by Ag deposition experiments due to the different $\mathrm{Ag}$ structures formed on both regions [fractal-like islands on clean patches vs rows of clusters on $R(15 \times 12)$ regions] (see also inset of Fig. 2). In most cases both the $R(15 \times 12)$ and the clean $(110)$ patches cover entire terraces. While wide terraces 

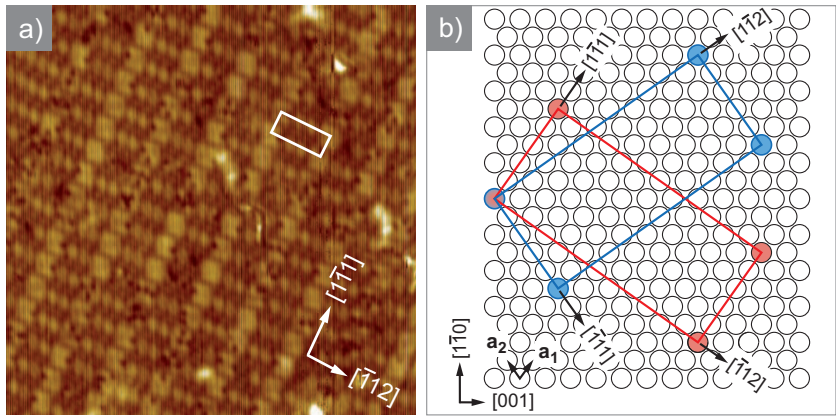

FIG. 1. (Color) (a) Typical STM image of $R(15$ $\times 12)$-C/W(110). Image size of $25 \times 25 \mathrm{~nm}^{2}$. (b) Schematic of the $R(15 \times 12)$ unit cell relative to the $\mathrm{W}(110)$ substrate. Unit cells of the two mirror domains are drawn in blue and red, respectively.

(>30 $\mathrm{nm})$ exhibit preferentially the $R(15 \times 12)$ structure, small terraces $(<30 \mathrm{~nm})$ are mostly clean, as can be seen from the statistical evaluation presented in Fig. 2. These data clearly indicate that the carbon content depends on the terrace width, which in turn implies that substrate steps assist the transport of carbon from the surface to the bulk. This finding is in agreement with the conclusions of Sunderland and Slavin, ${ }^{24}$ who found in their kinetic AES studies that carbon transport to the bulk occurs preferentially at the perimeters of carbon-covered patches. From the present STM data (e.g., inset of Fig. 2), it is obvious that the carbon patch boundaries can be identified with substrate steps.

\section{B. Deposition of silver}

\section{Low Ag coverages $(\theta \leq 0.12 M L)$}

Figure 3 shows STM images of the $R(15$ $\times 12)-\mathrm{C} / \mathrm{W}(110)$ surface, onto which low Ag coverages $(<0.12 \mathrm{ML})$ were deposited at 350-400 K. Small Ag clusters of monolayer height $(2.5-3.0 \AA)$ are observed, arranged on the rectangular grid given by the $R(15 \times 12)-\mathrm{C} / \mathrm{W}(110)$ template. Clusters are located in the depressions of the $R(15 \times 12)$ pattern which we associate with clean $\mathrm{W}(110)$ areas in the unit cell-as already discussed above. Between clusters of neighboring rows, the two characteristic faint maxima are observed [inset of Fig. 3(a)]. At coverages around 0.1 ML, chains of clusters are formed. These chains are running both along [1 $1 \overline{1}]$ and $[\overline{1} 11]$ due to the existence of two $R(15 \times 12)$ domains. At the ideal coverage of $\approx 0.12 \mathrm{ML}$, virtually all unit cells are filled with Ag clusters. Since the $(15 \times 12)-C / W(110)$ unit cell spans the area of 60 $\mathrm{W}$ surface atoms, this implies an average cluster size of $\approx 0.12 \times 60=7.2$ Ag atoms. Within the accuracy/ reproducibility of our coverage calibration, this number is compatible with cluster sizes of six to eight atoms. However, strong evidence for formation of $\mathrm{Ag}$ heptamers comes from the observed cluster shape. As can be seen from the edgeenhanced image in Fig. 3(b), most clusters appear to be quasihexagonal. The hexagonal shape of the clusters is most pronounced at the base of the clusters as shown in Fig. 3(d). While the red regions with heights beyond $200 \mathrm{pm}$ appear more or less rounded, the flanks (yellow) and the base (dark

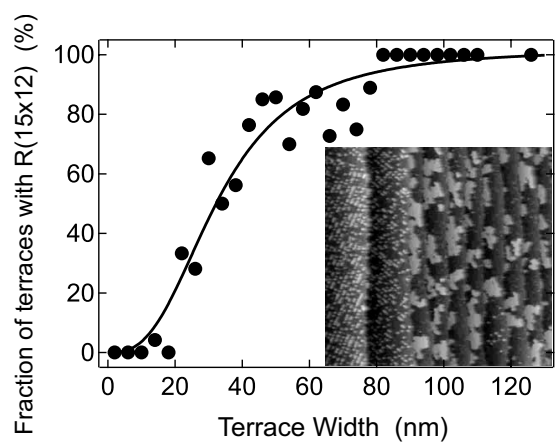

FIG. 2. Fraction of $R(15 \times 12)$ terraces as a function of terrace width. Data are obtained from Ag covered surfaces $(\theta<0.3 \mathrm{ML}$, $T<650 \mathrm{~K})$, where $R(15 \times 12)$ regions can easily be recognized due to the characteristic decoration with cluster rows. Inset: example of one of the images evaluated in order to obtain the depicted distribution curve.

green) of the clusters show a clear hexagonal shape. We conclude that the Ag heptamers consist of a quasihexagonal sixatom ring with the seventh $\mathrm{Ag}$ atom located in the center, as depicted in the ball model superimposed on the STM image
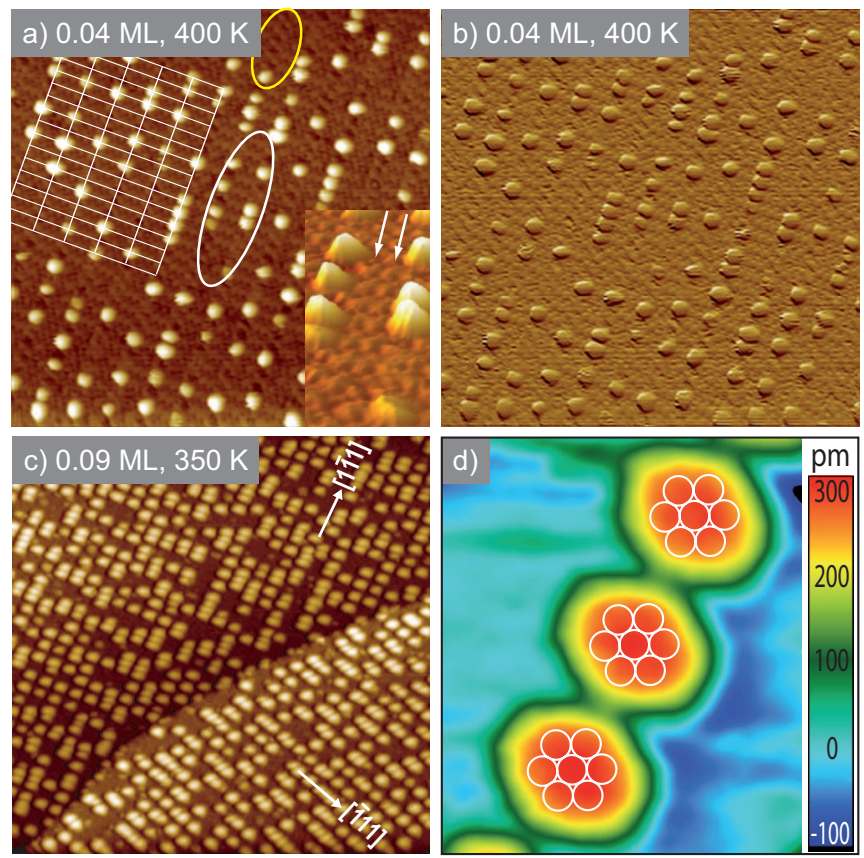

FIG. 3. (Color) STM images of small Ag coverages $(<0.12 \mathrm{ML})$ on $(15 \times 12) \mathrm{C}-\mathrm{W}(110)$. In (a) the periodicity of the underlying template is resolved. The inset in (a) shows an enlarged three-dimensional (3D) view of the region encircled in white. Due to the $\mathrm{C}(15 \times 12)$ template, two characteristic rows of small protrusions (indicated by arrows) can be seen between Ag clusters of neighboring chains. Ag clusters nucleate at sites imaged as depressions under the present tunneling conditions (see region encircled in yellow). Image (b) is the same as image (a) after image processing for edge enhancement to emphasize the hexagonal shape of the clusters. (c) Close to the ideal coverage, regular rows of clusters are formed. (d) Enlarged view with superimposed model of the heptamer clusters. Image sizes: [(a) and (b)] $40 \times 40$, (c) $80 \times 80$, and (d) $3.4 \times 4.0 \mathrm{~nm}^{2}$. 
of Fig. 3(d). Note that the size of the red, high regions of the Ag clusters is only slightly larger than the heptamer model, further supporting the assigned cluster size. Close inspection of the clusters on well-resolved images reveals that most ( $\approx 80 \%$ ) of the clusters exhibit about equal size: $5 \%-10 \%$ appear to be larger while $10 \%-15 \%$ are smaller. Thus, although we lack atomic resolution on the clusters, we infer that the size distribution is very narrow and that mostly clusters with a magic size of seven atoms are formed in the coverage range up to $0.12 \mathrm{ML}$.

\section{2. "High" Ag coverages $(\theta>0.12 M L)$}

At coverages beyond the optimal coverage of $0.12 \mathrm{ML}$, the morphology depends strongly on deposition temperature. On wide terraces $(>30 \mathrm{~nm})$ at $T \approx 350 \mathrm{~K}$, atoms in excess of $0.12 \mathrm{ML}$ agglomerate to larger islands of double-layer height which partially overgrow the cluster chains, visible as faint lines running along [111], i.e., diagonally from top left to bottom right in Fig. 4(a). The immediate formation of double layer rather than monolayer islands clearly indicates that bonding to the substrate is weak in the regions between the clusters. On narrow terraces $(<30 \mathrm{~nm})$ a different morphology is observed. Here large Ag islands but hardly any Ag-cluster chains can be discerned (see also Fig. 2). The morphology of these islands very much resembles that of $\mathrm{Ag}$ islands on clean W(110) two monolayer high. ${ }^{25}$ Thus we conclude that narrow terraces essentially consist of clean $\mathrm{W}(110)$.
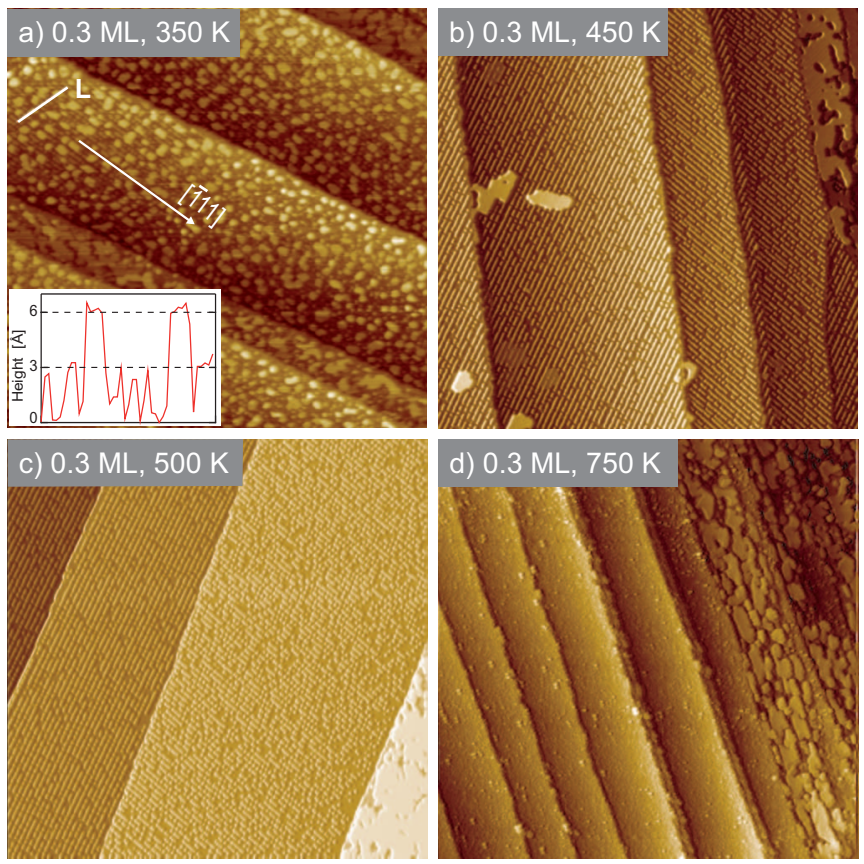

FIG. 4. (Color) STM images $\left(250 \times 250 \mathrm{~nm}^{2}\right)$ of $0.3 \mathrm{ML}$ $\mathrm{Ag} / R(15 \times 12)-\mathrm{C} / \mathrm{W}(110)$ for various deposition temperatures. In (a) note the existence of cluster rows running along [111], which are only visible as faint lines underneath the prominent $\mathrm{Ag}$ islands. The cluster rows are more discernable in the height profile along line $\mathrm{L}$, where they appear as narrow protrusions $\approx 3 \AA$ in height. The wide, $6 \AA$ high protrusions are due to double-layer Ag islands overgrowing the cluster rows.
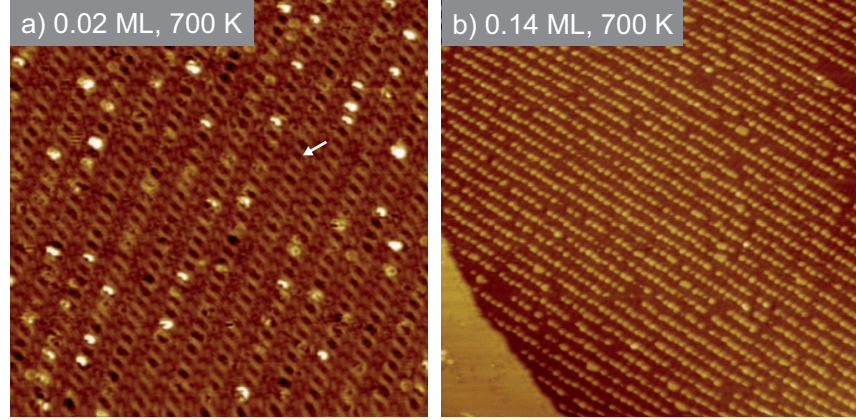

FIG. 5. (Color) STM images of small Co coverages deposited at $700 \mathrm{~K}$ on $R(15 \times 12) \mathrm{C}-\mathrm{W}(110)$. Image sizes: (a) $40 \times 40$ and (b) $80 \times 80 \mathrm{~nm}^{2}$.

Figures 4(b) and 4(c) show STM images for the same coverage of $\theta=0.3$ ML but at elevated deposition temperatures. At $T_{\mathrm{dep}}=450 \mathrm{~K}$ large regions with well-ordered cluster chains are obtained. In contrast to deposition at $350 \mathrm{~K}$, only few islands overgrowing the $\mathrm{Ag}$ nanodot chains are observed. Although these islands are larger than the corresponding ones at $350 \mathrm{~K}$, their volume is by far not sufficient to store all excess $\mathrm{Ag}$, i.e., $\mathrm{Ag}$ atoms beyond the ideal coverage of 0.12 ML. Instead the excess $\mathrm{Ag}$ is found on clean narrow terraces as, e.g., visible in the upper right corner of Fig. 4(b). There, nearly complete silver monolayers and bilayers were formed. At $500 \mathrm{~K}$ basically all excess $\mathrm{Ag}$ is found on such terraces [Fig. 4(c), bottom right corner]. Obviously at temperatures of $450 \mathrm{~K}$ or above, $\mathrm{Ag}$ atoms are quite mobile. They can diffuse over large distances and are even able to cross substrate steps. As Ag is mostly found on
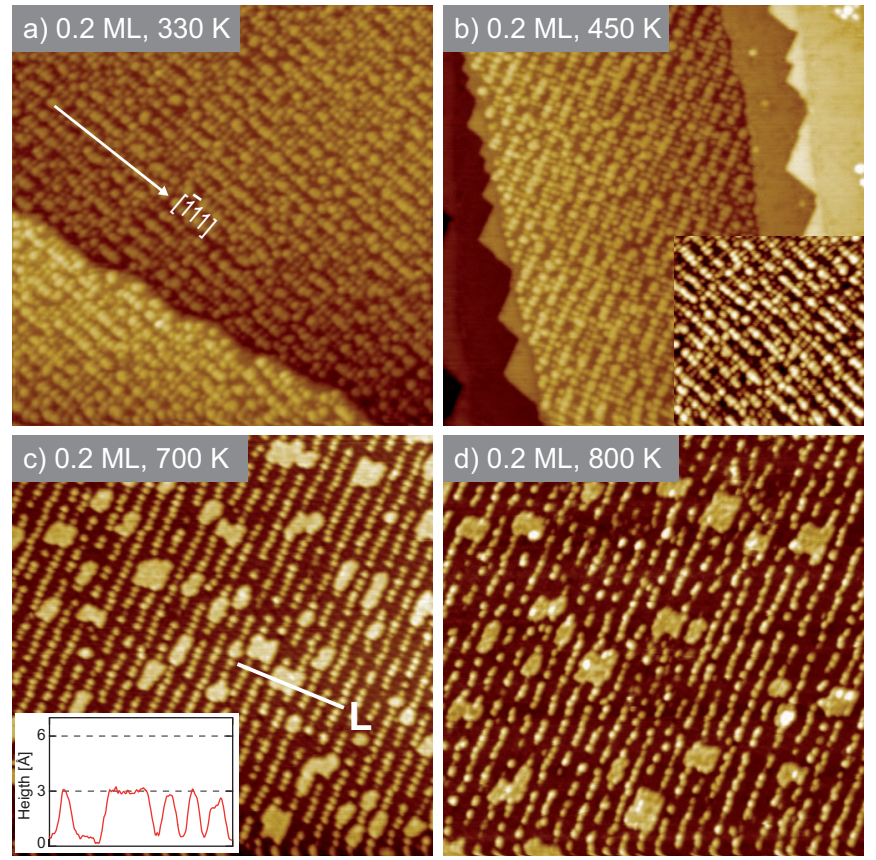

FIG. 6. (Color) STM images $\left(80 \times 80 \mathrm{~nm}^{2}\right)$ of $\approx 0.2 \mathrm{ML}$ $\mathrm{Co} / R(15 \times 12)-\mathrm{C} / \mathrm{W}(110)$ for various deposition temperatures. The inset in (b) shows an image from the second domain while the inset in (c) shows the profile along line L. Both clusters and islands are of monolayer height $(\approx 3 \AA)$. 
clean narrow terraces, this provides additional evidence that excess Ag is only weakly bound on $(15 \times 12)$ patches while the adsorption strength of the heptamer clusters seems to be roughly comparable with that on clean tungsten. This gives further support for our previous conclusion that Ag clusters nucleate in the clean regions of the $(15 \times 12)$ unit cell.

After deposition at $750 \mathrm{~K}$, ordered Ag-cluster chains are no longer found [Fig. 4(d)]. Most of the deposited material is then concentrated on narrow terraces. At this deposition temperature Ag atoms are so mobile during deposition that basically all of them diffuse to clean terraces where large islands can be formed and where slightly stronger binding sites (as compared to the cluster nucleation site) are available.

\section{Deposition of cobalt}

\section{Low Co coverages $(\theta \leq 0.14 M L)$}

STM images of low coverages of cobalt deposited at 700 $\mathrm{K}$ are shown in Fig. 5. At $\theta=0.02 \mathrm{ML}$ individual clusters can be discerned together with the underlying $R(15 \times 12)$ structure [Fig. 5(a)]. Obviously the Co clusters are located at the same positions within the $R(15 \times 12)$ unit cell as the $\mathrm{Ag}$ clusters [Fig. 3(a)]. However, in contrast to Ag, the clusters are less regularly sized. With increasing coverage more clusters nucleate and cluster sizes seem to become more regular until at the ideal coverage of $\theta \approx 0.14$ ML virtually all template unit cells are decorated with Co clusters. The cluster sizes are fairly homogeneous although they are less well defined than in the case of Ag. Furthermore it seems that cluster centers are not strictly located along straight lines but exhibit a small waviness. By and large, however, the behavior is similar to that of silver. Note that also the ideal coverages of 0.12 and $0.14 \mathrm{ML}$, respectively, agree with each other within our specified accuracy $( \pm 15 \%)$.

\section{High Co coverages $(\theta>0.14 M L)$}

As for silver the observed morphology depends strongly on temperature for coverages beyond the ideal one. However, for cobalt some remarkable differences are observed. At low deposition temperatures [330 K, Fig. 6(a)], additional small clusters appear. As the additional clusters exhibit no clear site preference and as cluster sizes vary considerably, the grid defined by the $R(15 \times 12)$ can only hardly be identified. All clusters are of monolayer height.

At $450 \mathrm{~K}$ ordering is improved both with respect to position and size of the clusters. Rows of "major" clusters are observed which exhibit the periodicity of the $R(15 \times 12)$ template. Between major clusters of neighboring rows, one or two "minor" clusters occur [inset of Fig. 6(b)]. These minor clusters are located at or close to the positions where the clean template exhibits the faint protrusions in the STM image [Fig. 1(a)]. At $700 \mathrm{~K}$ nice rows of regularly sized clusters are visible [Fig. 6(c)]. Thus this is the best temperature for deposition of ordered Co clusters. Excess cobalt atoms accumulate in monolayer-high islands connecting previously separated clusters (cluster coalescence). Finally at a deposition temperature of $T=800 \mathrm{~K}$, cluster ordering (both in position and size) fades out while larger islands of monolayer height still exist on the same terrace.

\section{DISCUSSION}

As already mentioned above, silver and cobalt exhibit similarities at low coverages. Both build well-ordered quasione-dimensional nanocluster arrays with the periodicity of the $R(15 \times 12)$ template. Clusters of both materials are anchored at the same site within the template unit cell. Not unexpectedly, the optimum temperature for formation of the cluster arrays is higher $(\approx 700 \mathrm{~K})$ for the less mobile Co atoms than for $\mathrm{Ag}(\approx 500 \mathrm{~K})$. The ideal coverages for formation of a full cluster array are 0.12 and $0.14 \mathrm{ML}$ for $\mathrm{Ag}$ and $\mathrm{Co}$, respectively, according to our coverage calibrations. At the ideal coverages the cluster size distributions appear to be narrow for both $\mathrm{Ag}$ and $\mathrm{Co}$ (as judged by eye) although the exact distribution is hard to quantify due to the small size of the clusters and the missing atomic resolution. Within the accuracy/reproducibility of our coverage calibrations $( \pm 15 \%)$, the values of both ideal coverages agree with each other. Thus it seems reasonable to assume that at the ideal coverage clusters of both metals contain the same number of atoms. The values of $0.12-0.14 \mathrm{ML}$ mentioned above correspond to average cluster sizes of $N=7-8$ atoms. The quasihexagonal shape observed for most silver clusters at all coverages up to $0.12 \mathrm{ML}$ provides strong evidence for formation of $\mathrm{Ag}$ heptamer clusters $(N=7)$. Where does the preference for this magic cluster size come from? In a recent work on Ag-cluster growth on the $\mathrm{Pb} / \mathrm{Si}(111)$ template, several magic sizes $(6,8,10,12, \ldots$ atoms $)$ were reported and explained by electronic shell closing. ${ }^{9}$ An alternative explanation is geometric closure due to the compact hexagonal shape of the heptamer clusters. However, although this concept is rather intuitive, geometric closure is expected to dominate only at larger cluster sizes. ${ }^{9}$ As only a single magic size rather than a sequence of magic numbers is observed in the present work, in our opinion the dominating effect stems from interactions with the substrate: modification of the W(110) substrate by carbon not only defines the preferred cluster nucleation sites but also "passivates" part of the unit cell, leaving only small regions where metal adsorption is favorable. The size of these small attractive regions then defines the preferred cluster size. In the present case the attractive regions are attributed to the clean parts of the unit cell, i.e., those parts which are only weakly perturbed by carbon. In particular the temperature-dependent Ag experiments at coverages beyond $0.12 \mathrm{ML}$ clearly demonstrate that $\mathrm{Ag}$ adsorption on areas in between the clusters is unfavorable and can only be stabilized at low temperatures, and even then only in islands of more than monolayer height. If temperature permits, silver atoms rather diffuse to clean $\mathrm{W}(110)$ terraces.

For cobalt the difference in adsorption strength between favorable and unfavorable areas seems to be less pronounced: at low temperatures $(T \leq 450 \mathrm{~K})$ clusters nucleate on "interstitial" sites and even at high temperatures $(\approx 700 \mathrm{~K})$, large islands cover unfavorable areas between the clusters at coverages beyond 0.14 ML. These islands are of monolayer height, pointing toward a stronger interaction with the substrate than in the case of silver, where only double-layer islands occur [compare insets of Figs. 4(a) and 6(c)]. As already pointed out above, we suppose that the less favorable areas in the unit cell exhibit higher carbon content. 
Thus we conclude that interaction of cobalt with carburized tungsten or even with carbon directly is considerably stronger than that for Ag. This conclusion is in agreement with several other observations: in contrast to silver, cobalt can form covalent bonds with carbon due to its partially unfilled $3 d$ orbitals. Furthermore Co forms metallic carbides, whereas for Ag only saltlike carbides exist. A further hint for a relatively strong interaction of Co with carburized tungsten comes from powder metallurgy, where Co is used as binder/ cement to obtain strong interconnections between tungstencarbide powder particles.

For the closely related system, $\mathrm{Au} / R(15$ $\times 12)-\mathrm{C} / \mathrm{W}(110)$ formation of nanodots was also reported by Varykhalov et al. ${ }^{13}$ although the Au nanodots in that work were substantially larger, less regularly spaced, and exhibit a broader size distribution. These differences may be attributed to a nonperfect template preparation, the relatively low deposition temperature $(300 \mathrm{~K})$, and larger coverage $(0.2 \mathrm{ML})$ used in the study. Similar to the present study, the Au nanoclusters show a tendency for alignment into one-dimensional rows although the ordering of the clusters is far from being perfect. However, the main difference between Au on the one side, and $\mathrm{Ag}$ and $\mathrm{Co}$ on the other side is the direction of the cluster rows. While, in our work, the Ag and Co clusters form rows along $[1 \overline{1} 1]$ and $[\overline{1} 11]$, respectively, i.e., along the short axis of the $R(15 \times 12)$ unit cells, the Au clusters align in rows along the long unit-cell axes, i.e., [112] and [112]. As the distance between neighboring $\mathrm{Au}$ cluster rows is $\approx 3 \mathrm{~nm}$, this would imply that cluster rows occur only on every second unit cell if the crystallographic directions given in Ref. 13 are correct. ${ }^{26}$ In view of the similarities between $\mathrm{Ag}$ and $\mathrm{Co}$, the differences to $\mathrm{Au}$ are quite surprising. The exploration and understanding of these differences remains an interesting task. After annealing films with $0.5 \mathrm{ML}$ of gold to high temperatures, $(1000 \mathrm{~K})$ Varykhalov et al. ${ }^{13}$ observed well-ordered arrays of nanodots on some patches of the $\mathrm{Au} / R(15 \times 12)-\mathrm{C} / \mathrm{W}(110)$ surface. However, it is not yet clear whether these nanodots are clusters or single Au atoms.
Interestingly, there are two of such nanodots in each $R(15$ $\times 12$ ) unit cell, again in contrast to adsorption of $\mathrm{Ag}$ and $\mathrm{Co}$.

\section{SUMMARY}

In summary, we showed that the $R(15 \times 12) \mathrm{C}-\mathrm{W}(110)$ surface forms a suitable template for creation of well-ordered chains of $\mathrm{Ag}$ and Co clusters on a rectangular grid of 3.10 $\times 1.37 \mathrm{~nm}^{2}$. The optimum preparation temperatures are around 500 and $700 \mathrm{~K}$, respectively. The cluster size in the present study is controlled with high precision. Although we lack atomic resolution, we estimate that for $\mathrm{Ag}$ about $80 \%$ of the clusters consist of seven silver atoms. We identify interactions with the substrate as the main reason for this preferred size. Due to the limited availability of strong binding sites within the $R(15 \times 12)$ unit cells, growth of larger clusters is highly unfavorable. The absence of smaller clusters can simply be attributed to the high deposition temperatures (350-700 K), where small clusters decompose during growth and only clusters beyond a critical size are stable. ${ }^{27}$

Apart from enabling fabrication of a uniquely welldefined cluster array, the $R(15 \times 12)-\mathrm{C} / \mathrm{W}(110)$ template offers another distinct advantage: namely, stability up to temperatures of more than $1000 \mathrm{~K}$. Thus it can also be used at elevated deposition temperatures to produce nanodot arrays of species even less mobile than cobalt. The present results clearly demonstrate that making use of the interplay of various interactions in a system allows a tuning of nanostructures with atomic precision. The challenge is the identification of the relevant interactions, and the proper choice of template and adsorbate materials to obtain the desired size and shape distributions.

\section{ACKNOWLEDGMENTS}

Financial support by the Austrian Science Fund (Grant No. S9004) and technical assistance by Reinhold Pramsoler are gratefully acknowledged.

\footnotetext{
*norbert.memmel@uibk.ac.at

${ }^{1}$ J. V. Barth, G. Costantini, and K. Kern, Nature (London) 437, 671 (2005)

${ }^{2}$ N. F. van Hulst, Nature (London) 448, 141 (2007), and references therein.

${ }^{3}$ H. Brune, Surf. Sci. Rep. 31, 121 (1998).

${ }^{4}$ D. D. Chambliss, R. J. Wilson, and S. Chiang, Phys. Rev. Lett. 66, 1721 (1991).

${ }^{5}$ J. A. Meyer, I. D. Baike, E. Kopatzki, and R. J. Behm, Surf. Sci. 365, L647 (1996).

${ }^{6}$ M. Y. Lai and Y. L. Wang, Phys. Rev. Lett. 81, 164 (1998).

${ }^{7}$ L. Vitali, M. G. Ramsey, and F. P. Netzer, Phys. Rev. Lett. 83, 316 (1999).

${ }^{8}$ J.-L. Li, J. F. Jia, X. J. Liang, X. Liu, J. Z. Wang, Q. K. Xue, Z. Q. Li, J. S. Tse, Z. Zhang, and S. B. Zhang, Phys. Rev. Lett. 88, 066101 (2002).

${ }^{9}$ Y. P. Chiu, L. W. Huang, C. M. Wei, C. S. Chang, and T. T.
}

Tsong, Phys. Rev. Lett. 97, 165504 (2006).

${ }^{10}$ N. Weiss, T. Cren, M. Epple, S. Rusponi, G. Baudot, S. Rohart, A. Tejeda, V. Repain, S. Rousset, P. Ohresser, F. Scheurer, P. Bencok, and H. Brune, Phys. Rev. Lett. 95, 157204 (2005).

${ }^{11}$ C. Didiot, S. Pons, B. Kierren, Y. Fagot-Revurat, and D. Malterre, Surf. Sci. 600, 3917 (2006).

${ }^{12}$ A. Varykhalov, C. Biswas, W. Gudat, and O. Rader, Phys. Rev. B 74, 195420 (2006).

${ }^{13}$ A. Varykhalov, O. Rader, and W. Gudat, Phys. Rev. B 77, 035412 (2008).

${ }^{14}$ A. T. N'Diaye, S. Bleikamp, P. J. Feibelman, and T. Michely, Phys. Rev. Lett. 97, 215501 (2006).

${ }^{15}$ Y. L. Wang and M. Y. Lai, J. Phys.: Condens. Matter 13, R589 (2001).

${ }^{16}$ E. Bauer, Surf. Sci. 7, 351 (1967).

${ }^{17}$ R. Baudoing and R. M. Stern, Surf. Sci. 10, 392 (1968). 
${ }^{18}$ In some publications the structures are also denoted as (15 $\times 3) R 14^{\circ}$ or $(15 \times 12) R 14^{\circ}$, respectively, although an angle of $14^{\circ}$ does not exist between the overlayer unit-cell axes and any of the low-index directions of W(110).

${ }^{19}$ S. D. Foulias, K. J. Rawlings, and B. J. Hopkins, J. Phys. C 14, 5403 (1981).

${ }^{20}$ K. J. Rawlings, S. D. Foulias, and B. J. Hopkins, J. Phys. C 14, 5411 (1981).

${ }^{21}$ J. G. Macmillan, A. J. Slavin, and K. J. Sunderland, Surf. Sci. 173, 138 (1986).

${ }^{22}$ M. Bode, R. Pascal, and R. Wiesendanger, Surf. Sci. 344, 185
(1995).

${ }^{23}$ A. Varykhalov, O. Rader, and W. Gudat, Phys. Rev. B 72, 115440 (2005).

${ }^{24}$ K. J. Sunderland and A. J. Slavin, Surf. Sci. 233, 89 (1990).

${ }^{25}$ C. Deisl, E. Bertel, M. Bürgener, G. Meister, and A. Goldmann, Phys. Rev. B 72, 155433 (2005).

${ }^{26}$ The crystallographic directions in Ref. 13 were deduced from the pattern of the $R(15 \times 3)$ unit cell, which was observed simultaneously on the surface. A. Varykhalov (private communication).

${ }^{27}$ J. A. Venables, G. D. T. Spiller, and M. Hanbücken, Rep. Prog. Phys. 47, 399 (1984). 